\title{
reviscafuences
}

ISSN: $1575-7072$ | e-ISSN: 2172-7775

Páginas: $90-103$

Recibido: 2021-02-16

Revisado: 2021-03-27

Aceptado: 2022-01-03

Preprint: 2022-01-03

Publicación Final: 2022-01-30

www.revistascientificas.us.es/index.php/fuentes/index

DOI: https://doi.org/10.12795/revistafuentes.2022.15258

\section{Análisis de la eficacia de los planes de formación profesional para el empleo desde el punto de vista del mercado laboral}

\section{Analysis of the effectiveness of vocational training plans for employment from the point of view of the labor market}

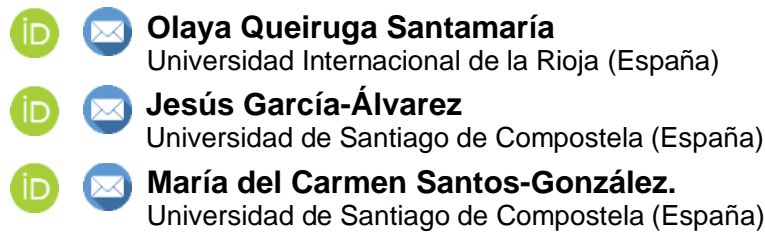

\section{Resumen}

La Formación Profesional para el Empleo (FPE) se define como aquella que responde a las necesidades del mercado laboral y se orienta a la adquisición, mejora y actualización continua de las competencias y cualificaciones profesionales, favoreciendo la formación a lo largo de toda la vida. Su finalidad es impulsar el desarrollo personal y profesional de las personas trabajadoras (ocupadas y desempleadas), al tiempo que promueve su empleabilidad. Considerando lo anterior, se presenta un estudio que responde al objetivo de determinar el conocimiento que poseen las empresas acerca de la oferta de formación profesional y los mecanismos que permiten su transferencia al puesto de trabajo. La metodología empleada es de corte cuantitativo, utilizando el cuestionario como instrumento de recogida de información ( $n=331$ empresas). Los resultados confirman, por un lado, el desconocimiento de las empresas acerca del funcionamiento, beneficios y oportunidades que ofrece la formación para el empleo, y por otro, la elevada valoración de las competencias de tipo transversal en la formación de los trabajadores/as, destacando su importancia para el diseño de los actuales planes de formación. Se concluye señalando la necesidad de una mayor difusión de las políticas activas de empleo centradas en la oferta de formación, así como la pertinencia de introducir las competencias transversales en su planificación.

\begin{abstract}
Vocational Training for Employment (VTE) is defined as that which give responds to the needs of the labor market and is oriented to the acquisition, improvement and continuous updating of professional competences and qualifications, promoting lifelong learning. Its purpose is to promote the personal and professional development of working people (employed and unemployed) while promoting their employability. Considering this, the objective is to determine the knowledge that companies possess in relation to the offer of professional training and the mechanisms that allow its transfer to the labor market. The methodology used is quantitative, using the questionnaire as an instrument for collecting information ( $n=331$ companies). The results confirm, on the one hand, the lack of knowledge of companies about the operation, benefits and opportunities offered by training for employment. On the other hand, it is confirmed the high value of soft skills in the training of workers, highlighting its importance for the design of current training plans. It concludes by pointing out the need for a greater dissemination of active employment policies focused on the training offer, as well as the relevance of introducing transversal competences in its planning.
\end{abstract}

\section{Palabras clave / Keywords}

Eficacia, competencias transversales, cualificación profesional, mercado laboral, planes de formación.

Effectiveness, soft skills, professional qualification, labour market, training plan. 


\section{Introducción}

La continua evolución del mercado laboral ha provocado que los puestos de trabajo y sus funciones hayan experimentado significativas transformaciones en los últimos años (Rial, 2002; Izquierdo, Puente, \& Font, 2013). En consecuencia, para desempeñar una ocupación ya no basta con adquirir, en un momento dado de nuestro ciclo vital, ciertos conocimientos técnicos y/o teóricos (Casal, Colomé, \& Comas, 2003; Lorente, 2011; Pineda-López \& Moreno-Bastida, 2019). Ahora, también es necesario poseer una serie de destrezas transversales, habilidades y aptitudes que puedan aplicarse a otros empleos y empresas, y que permitan resolver los problemas profesionales de forma autónoma y flexible. Es lo que Martínez-Clares, GonzálezLorente y Rebollo-Quintela (2019) denominan competencias para la empleabilidad.

En esta línea, la empleabilidad se ve condicionada por las competencias y cualificaciones que facilitan y promueven la transferencia, así como por la capacidad de los sujetos para aprovechar las oportunidades de educación y de formación que se les presentan. Es así como se reconoce la importancia que tiene la formación para el desarrollo de las competencias transversales o soft skills. De esta suerte, y considerando el actual paradigma socioeconómico, disponer de competencias transversales -a menudo denominadas "competencias transferibles", "competencias clave", o "competencias de empleabilidad"- contribuye en buena medida a la consecución de una carrera profesional estable y, en definitiva, a la promoción de la empleabilidad individual (Archer \& Davison, 2008; Bennett, 2002; Blair \& Deming, 2020; Bridgstock, 2009; Deming, 2017; Suleman, 2018; World Economic Forum, 2018). Y es que, la elevada valoración de las competencias transversales sugiere una modificación en los procedimientos de selección, que han dejado de focalizarse en la evaluación de cualificaciones y competencias técnicas, vinculadas a una determinada ocupación, para centrarse en la valoración pormenorizada de las disposiciones y capacidades individuales. Por ello, la formación o aprendizaje a lo largo y ancho de la vida, entendida como toda actividad formativa realizada en cualquier momento del ciclo vital con el fin de mejorar las competencias y/o cualificaciones (Ministerio de Educación y Formación Profesional, 2020), constituye la estrategia idónea para favorecer la adaptación a los nuevos cambios en el mundo del trabajo.

Lo cierto es que nos encontrarnos en un escenario altamente competitivo y en constante evolución, donde las empresas demandan un capital humano en permanente actualización y reciclaje, que afronte los retos tanto a nivel de competencias técnicas como de competencias transversales (Valencia, 2005). La estrategia a asumir parece clara, ya que las apuestas se inclinan hacia la formación como la alternativa más adecuada para dar respuesta a estas necesidades y demandas (Pineda, 2002; Pérez Esparells, \& Rahona, 2009, Soler, 2014). Sin embargo, ha de tenerse en cuenta que no se trata simplemente de formar a todo aquel que carece de cualificación profesional. Antes de llevar a cabo planes y programas de formación dirigidos a la adquisición o mejora de cualificación profesional de las personas trabajadoras (ocupadas y/o desempleadas) resulta indispensable investigar y analizar cuáles son las necesidades y demandadas del mercado de trabajo: qué, cómo, dónde y cuándo lo necesitan (Marhuenda, 2012; Ros, Navas, \& Marhuenda, 2017). Para que la formación de oferta concuerde con las demandas del tejido empresarial, debe existir comunicación entre el ámbito educativo/formativo y el laboral, pues de lo que se trata es de que ambos, que comparten intereses comunes, puedan avanzar en la misma dirección.

Por este motivo, la formación profesional, en sus diferentes modalidades (inicial y para el empleo), se ha visto potenciada en los últimos años. La situación de crisis económica que todavía afecta a nuestra sociedad, junto a la necesidad de asumir un modelo productivo más flexible, ha motivado una apuesta por la formación como estrategia ante las necesidades de trabajadores/as y empresas (Calvo, Payá, \& Sigalat, 2021).

Como se recoge en sus lineamientos, la Formación Profesional para el Empleo (FPE) tiene como objetivo principal la formación y capacitación de las personas para su acceso al empleo y permanencia, actualizando sus conocimientos y competencias. Las iniciativas de formación que se llevan a cabo desde este subsistema y que se desarrollan en el marco del Sistema Nacional de Empleo (SNE), están dirigidas a la adquisición, mejora y actualización continua de las competencias y cualificaciones profesionales, favoreciendo la formación permanente de la población activa y conjugando las necesidades de las personas, de las empresas, de los territorios y de los sectores productivos (Ministerio de Educación y Formación Profesional, 2021). En palabras de Osuna et al. (2010), es así como la formación profesional para el empleo constituye la iniciativa idónea para hacer frente al desempleo.

En cuanto a la evaluación de la FPE, es necesario subrayar que se lleva a cabo a través de los planes anuales de evaluación de la calidad, impacto, eficacia y eficiencia del conjunto del sistema de FPE en el ámbito laboral, coordinado por el Servicio Público de Empleo Estatal (SEPE), con el apoyo y participación de los órganos o entidades competentes de las comunidades autónomas y de las organizaciones empresariales y sindicales 
más representativas. La finalidad de estos planes de evaluación es contemplar y observar los fines del propio sistema.

En el contexto de este trabajo, nos preocupa especialmente el apartado que se refiere a la atención a los requerimientos del mercado de trabajo y a las necesidades de las empresas, pues busca proporcionar a los trabajadores/as las competencias, conocimientos y prácticas adecuadas. En el informe Formación Profesional para el Empleo del año 2016, publicado por el SNE, se analizan diferentes dimensiones, de las que llama especialmente la atención la centrada en la detección de necesidades formativas por parte de los agentes del SNE que contemplen de forma integral las prioridades de empresas y trabajadores/as en cada sector económico y territorio. En este análisis se concluye que los procesos para determinar las prioridades formativas que son incorporadas en las diferentes modalidades de formación de oferta presentan debilidades que limitan su eficacia como sistema integral y participativo de detección de necesidades de formación en empresas y trabajadores/as (SNE, 2016). Además, se deja constancia en el mismo informe de que la escasa coherencia entre el Subsistema de FPE y el resto de recursos y procesos de inserción laboral (orientación, intermediación, asesoramiento en fórmulas de autoempleo, entre otras) que son desplegados por los servicios públicos de empleo en colaboración con otras entidades especializadas en la intervención sociolaboral y en la prestación de servicios de recursos humanos a empresas y organizaciones (Queiruga, 2018).

Amén de ello, se plantea el presente estudio, en el que se analiza el conocimiento que poseen las empresas acerca de la formación profesional para el empleo y sus mecanismos para lograr la transferencia al mercado de trabajo, con especial énfasis en la valoración que realizan de las competencias de carácter transversal habida cuenta de su importancia para el mundo laboral actual.

Partiendo de estos objetivos, y tras abordar los aspectos clave relacionados con el ajuste de la FPE a las demandas del mercado laboral, se describen los datos identificativos de las empresas que participan en el estudio en función del ámbito profesional, tamaño y provincia en la que tienen registrado su domicilio social. A continuación, se analiza la dimensión relacionada con el conocimiento del subsistema de FPE, del Catálogo Nacional de Cualificaciones Profesionales (CNCP) y de los Certificados de Profesionalidad (CP). Para finalizar, se identifican las necesidades de formación vinculadas a las competencias que demandan las empresas a sus trabajadores/as, así como la percepción que tienen en relación con la evolución del mercado de trabajo para las ocupaciones de su ámbito laboral.

A tal efecto, se ha podido demostrar un elevado desconocimiento de las organizaciones en lo que respecta al sentido y funcionamiento del subsistema de FPE, obligando, por tanto, a pensar en una mejor y más eficaz dotación de herramientas que aumenten su proyección y dinamización en el entorno productivo.

\section{Ajuste de la formación profesional para el empleo a las demandas del mercado laboral}

Antes de analizar la importancia de la formación como estrategia para la mejora de la empleabilidad, es fundamental clarificar ciertos términos relacionados con la FPE. Tal y como indica el propio CEDEFOP (2014), la cualificación es el resultado formal (certificado, diploma o título) de un proceso de evaluación y validación, obtenido cuando una administración competente determina que una persona posee los resultados de aprendizaje correspondientes a una norma dada y/o posee las competencias necesarias para ejercer un empleo en un entorno de actividad profesional específica. Así, la cualificación como requisito para el empleo, se refiere a los conocimientos, destrezas y aptitudes necesarias para desempeñar las tareas asociadas a un puesto de trabajo concreto (Bunk, 1994; Olaz, 2011).

Por otro lado, cuando hablamos de FPE, inevitablemente hacemos referencia al mercado laboral, ya que esta, como su nombre indica, va dirigida al empleo, al trabajo y a mejorar las competencias profesionales que permiten dar respuesta a las nuevas necesidades que van surgiendo.

Sobre el ajuste de la formación a las demandas del mercado laboral, Rodríguez Sánchez (2002) subraya que el vínculo entre la formación profesional y la realidad laboral y productiva es un factor tremendamente determinante a la hora de decidir las necesidades formativas (Buendía, \& Berrocal, 2008; Buendía, Expósito, \& Sánchez, 2012; Ros et al., 2017; Fernández-García, García Llamas, \& García Pérez, 2019). Las relaciones laborales han evolucionado de manera significativa, sobre todo en la última década. Aquella aspiración de todo trabajador por conseguir un puesto de trabajo "para toda la vida", es decir, un puesto seguro en una organización sólida y estable, ha dejado paso a un ideal totalmente obsoleto. La realidad actual impone una concepción completamente contraria.

Las organizaciones actuales buscan equipos de trabajo flexibles, altamente cualificados y con capacidad de adaptación a los cambios y de asumir reconversiones de forma continuada. Por ello, la formación de calidad 
no solo debe ofrecer conocimientos técnicos/teóricos, sino también responder a las necesidades productivas de cada puesto de trabajo, produciéndose una armónica simbiosis entre los ámbitos educativo/formativo y laboral.

Sin embargo, como bien indican Chisvert y Marhuenda (2012), en España contamos con un "sistema productivo inadecuado, mal orientado, y de larga tradición y cultura empresarial en la que no se ha potenciado suficientemente la formación ni la cualificación profesional" (p. 164). Por ello y, en consecuencia, la estructura de cualificaciones de la población activa es inversa a la existente en Europa, ya que contamos con

\begin{abstract}
una amplia base de personas sin ninguna cualificación, un techo amplio de personas con titulación universitaria, y una franja central muy reducida de personas tituladas en formación profesional. Esta tendencia no se va a interrumpir mediante la invitación más reciente a asemejar e introducir en España un sistema similar al alemán; ya que se sigue pensando el problema como algo propio del sistema educativo y no del sistema productivo; no es la cultura escolar, sino la empresarial, la que está consintiendo y alentando esa estructura de cualificaciones invertida (Chisvert y Marhuenda, 2012, p. 164).
\end{abstract}

Además, pese a que existe un elevado porcentaje de personas trabajadoras desempleadas, y a la inversión tan elevada que se está destinando a la cualificación profesional de este colectivo, las empresas manifiestan que tienen serias dificultades para cubrir sus necesidades. En estos términos, se observa un problema de sincronización entre las demandas de capacitación del mercado laboral y la oferta formativa. Por ello, cabe formular sobre esta cuestión varias preguntas, ¿se llevan a cabo análisis rigurosos para contribuir a un mayor ajuste entre las dimensiones formativa y laboral? ¿por qué no se estudia lo suficiente a las empresas y sus necesidades? ¿por qué no se planifica formación para la inserción laboral? La respuesta puede parecer evidente. Sin embargo, a pesar de que las empresas están demandando continuamente unos perfiles determinados, se sigue ofertando formación en ocupaciones que apenas tienen inserción laboral (Servicio Público de Empleo Estatal, 2019a, 2019b).

En esta misma línea, el resumen ejecutivo Trabajar en 2033, presentado por PwC España (2014), informa de una serie de recomendaciones a las Administraciones Públicas para los próximos años relacionadas con el fortalecimiento de lazos entre el ámbito educativo/formativo y laboral, indicando que debe disminuirse notablemente el desajuste existente entre oferta y demanda. Para alcanzar este objetivo, señalan que es necesario

alcanzar un acuerdo de Estado en educación; fomentar la sincronía y colaboración entre instituciones educativas y empresas; orientar a los jóvenes desde edades tempranas en el ámbito profesional; potenciar un cuerpo docente de calidad y próximo a la realidad del mercado; poner en valor la Formación Profesional; consolidar la formación dual, mejorar la eficacia de los servicios de colocación y empleo; y potenciar la formación continua a lo largo de la vida laboral (p.13).

Así pues, la evidencia refiere la importancia de reflexionar sobre hacia dónde debe dirigirse la atención para responder a las necesidades del mercado laboral, incluyendo las de empresas y trabajadores/as (ocupados/as y desempleados/as). A su vez, se antoja pertinente una reforma del sistema educativo y laboral para que ambas esferas discurran en paralelo y apuesten por una mayor orientación dirigida a trabajadores/as y empresas. Y es igualmente conveniente, realizar estudios prospectivos que permitan definir las líneas que guiarán la formación de futuro, tanto a medio como a largo plazo.

\title{
3. Metodología
}

El trabajo que aquí se presenta sigue una metodología de corte cuantitativo, aunque recoge también aspectos cualitativos e interpretativos. Así, y al tratarse de una investigación en la que se estudia la adaptación de la formación a los requerimientos y necesidades del mercado laboral, se ha considerado adecuado seguir un análisis fundamentalmente descriptivo y comparativo. Por su parte, el estudio ex-post facto resulta ser el más pertinente, dado que esta investigación no se orienta al conocimiento de variables dependientes explicativas, sino a la descripción y caracterización de las mismas.

\subsection{Instrumento}

Como técnica de recogida de información se ha utilizado la encuesta en forma de cuestionario que presenta, por un lado, un componente descriptivo (proporciona información respecto del perfil de los participantes), y 
por otro, un componente interpretativo (se buscan comparaciones entre subgrupos en función de la familia profesional y el tamaño de la empresa) para analizar posibles diferencias y determinar la estructura de ciertas variables de interés.

A través de su aplicación se pretende conocer si las diferentes empresas de la Comunidad Autónoma de Galicia del sector de la construcción y afines (edificación y obra civil; electricidad y electrónica; energía y agua; madera, mueble y corcho; e industrias extractivas) conocen el subsistema de FPE y el contexto en el cual se materializan sus principios y funciones. También es objeto de estudio en este trabajo la evaluación de sus necesidades, analizando la valoración que realizan de las competencias transversales en la formación de sus trabajadores y trabajadoras.

El cuestionario se articula en torno a cinco grandes bloques. El primero de ellos recoge datos de identificación (provincia, familia profesional y tamaño de empresa). El segundo, incluye datos referidos al conocimiento del subsistema de FPE, del CNCP y de los CP. Esta cuestión actúa de filtro sobre el tercero de los bloques, en el cual se busca determinar si las empresas tienen o han tenido contratado o en prácticas a algún trabajador/a que realizase un CP, así como la adecuación de la oferta formativa existente a los requerimientos del mercado laboral. En el cuarto bloque, se analiza la importancia concedida a las competencias de carácter transversal en el perfil profesional de sus trabajadores/as a través de una escala de intervalo. $Y$, por último, en el quinto de ellos, se formulan cuestiones de tipo prospectivo sobre la evolución de las necesidades en su sector de actuación.

Dado que el instrumento fue elaborado ad hoc para esta investigación, se tuvo que proceder a su validación. Concretamente, para su diseño se organizaron varias sesiones de trabajo individualizadas con personal experto en el ámbito de la formación profesional y de las cualificaciones (14 jueces expertos). Este sistema de consulta y las revisiones efectuadas permitieron dotar al instrumento de un alto grado de validez.

En relación con la escala de competencias transversales, para conocer su fiabilidad se recurrió al cálculo de la consistencia interna a través del coeficiente Alpha de Cronbach ( $\alpha$ ). Se presentan a continuación los ítems que la configuran en un intervalo que varía de 1 (poco valorada) a 5 (muy valorada) (ver tabla 1).

Tabla 1

Escala. Competencias transversales

\begin{tabular}{ll}
\hline Código & \\
\hline C1 & Capacidad de trabajo en equipo \\
C2 & Uso efectivo del tiempo \\
C3 & Capacidad de resolución de problemas \\
C4 & Preocupación por la calidad y la mejora \\
C5 & Compromiso \\
C6 & Capacidad para adquirir nuevos conocimientos con rapidez \\
C7 & Capacidad de organización y planificación \\
C8 & Adaptación a los cambios \\
C9 & Polivalencia \\
C10 & Motivación por alcanzar metas \\
C11 & Autonomía \\
C12 & Capacidad para tomar decisiones \\
C13 & Habilidades interpersonales \\
C14 & Motivación por el trabajo \\
C15 & Capacidad para rendir bajo presión \\
C16 & Mediación de conflictos \\
C17 & Espíritu emprendedor \\
\hline
\end{tabular}

A este respecto, los resultados informan de una elevada fiabilidad, donde $\alpha$ es igual a ,828; lo que permite afirmar que el instrumento tiene buena consistencia interna (Acosta-Banda, Aguilar-Esteva, Patiño Ortiz, \& Patiño Ortiz, 2021; Cronbach, 1951).

\subsection{Procedimiento}

La aplicación del instrumento se llevó a cabo entre diciembre de 2018 y enero de 2020. En un primer momento, se elaboró el cuestionario en soporte papel para aplicarlo de forma presencial $(n=36)$, telefónicamente $(n=137)$ o por correo electrónico $(n=71)$. Sin embargo, a partir de julio de 2019 , la aplicación también se realizó a través de una plataforma de encuestas en línea $(n=87)$. En esta diversificación de alternativas para la 
cumplimentación del instrumento se evitaron posibles duplicidades. De esta forma, se obtuvo una mayor participación, que finalmente alcanzó un $71.5 \%$ del total de muestra seleccionada.

Los datos recogidos en la aplicación de los cuestionarios han sido tratados con el paquete estadístico IBM SPSS Statistics versión 24.

\subsection{Participantes}

La población fue determinada en base a todas las empresas pertenecientes al CNAE de construcción (grupo F) que llevan a cabo su actividad en Galicia. Según el Instituto Gallego de Estadística (IGE, 2020), en el año 2018, había 31.350 empresas (16.018 personas físicas, 437 sociedades anónimas, 13.013 sociedades de responsabilidad limitada y 118 sociedades cooperativas), tal y como se muestra en la tabla 2 . No obstante, si se toman como base los epígrafes 41 (construcción de edificios), 42 (ingeniería civil) y 43 (actividades de construcción especializada), según el Observatorio de la Fundación Laboral de la Construcción (2020), cuyo análisis es más preciso y riguroso al incluir el registro actualizado de los datos de las empresas que forman parte del Convenio General del Sector de la Construcción, estaríamos hablando de 29.686 empresas, cifra muy similar a la que aporta el IGE.

\section{Tabla 2}

Empresas con actividad en Galicia (CNAE: Construcción - grupo F)

\begin{tabular}{clc}
\hline Año & & $\mathbf{2 0 1 8}$ \\
\hline & La Coruña & 12.849 \\
Provincia & Lugo & 4.136 \\
& Orense & 4.180 \\
& Pontevedra & 10.185 \\
\hline C. Autónoma & Galicia & 31.350 \\
\hline
\end{tabular}

La técnica de muestreo utilizada ha sido no probabilística estratificada, ya que se ha seleccionado una muestra representativa de las empresas que constan en Galicia por familias y áreas profesionales en diferentes bases de datos, entre las que destaca Construmatica. A partir de los resultados obtenidos se han consultado datos de contacto a través de diferentes buscadores de información para, finalmente, conformar una muestra de empresas que:

- Solicitaron el pase presencial del cuestionario.

- Respondieron al cuestionario en la misma llamada o fijaron una fecha para participar.

- Cumplimentaron el cuestionario en línea.

- Cubrieron y enviaron el cuestionario a través del correo electrónico.

De esta forma, la muestra productora de datos $(n=331)$ se distribuye del siguiente modo: 228 de edificación y obra civil, 43 de energía y agua, 52 de electricidad y electrónica, 3 de industrias extractivas y 5 de madera, mueble y corcho. En cuanto a su perfil, está formada por 206 representantes legales, 29 responsables de recursos humanos y 96 tutores/as de prácticas con diferente formación.

Para el análisis de los datos obtenidos se han utilizado tanto pruebas paramétricas como no paramétricas. En primer lugar, se sometieron las puntuaciones a análisis de respuesta múltiple, a fin de conocer la distribución general de las puntuaciones asignadas por las empresas a los distintos aspectos de forma conjunta para, posteriormente, realizar análisis de tipo descriptivo y comparativo.

\subsection{Características de las empresas participantes}

En la tabla 3 se observa la distribución de la muestra de empresas participantes por provincias, familia profesional y tamaño de empresa. 
Tabla 3

Descripción de las empresas participantes

\begin{tabular}{|c|c|c|c|}
\hline & & Frecuencia & Porcentaje \\
\hline \multirow{4}{*}{ Provincia } & A Coruña & 138 & 41.7 \\
\hline & Lugo & 58 & 17.5 \\
\hline & Ourense & 48 & 14.5 \\
\hline & Pontevedra & 87 & 26.3 \\
\hline \multirow{5}{*}{$\begin{array}{l}\text { Familia } \\
\text { profesional }\end{array}$} & Edificación y obra civil & 228 & 68.9 \\
\hline & Electricidad y electrónica & 52 & 15.7 \\
\hline & Energía y agua & 43 & 13 \\
\hline & Madera, mueble y corcho & 5 & 1.5 \\
\hline & Industrias extractivas & 3 & 0.9 \\
\hline \multirow{4}{*}{$\begin{array}{l}\text { Tamaño de la } \\
\text { empresa }\end{array}$} & Microempresa (<10 trabajadores/as) & 169 & 51.1 \\
\hline & Pequeña empresa (10-30 trabajadores/as) & 94 & 28.4 \\
\hline & Mediana empresa (31-100 trabajadores/as) & 46 & 13.9 \\
\hline & Gran empresa (más de 100 trabajadores/as) & 22 & 6.6 \\
\hline
\end{tabular}

La mayoría de las empresas participantes tiene su domicilio social en la provincia de A Coruña (41.7\%) y Pontevedra (26.3\%). Casi el $70 \%$ de las empresas que componen la muestra participante forman parte de la familia profesional de edificación y obra civil, el $15.7 \%$ de electricidad y electrónica, el $13 \%$ de energía y agua, el $1.5 \%$ pertenecen a la familia profesional de madera, mueble y corcho y el $0.9 \%$ a la de industrias extractivas. Por último, también se observa que la mayoría de las organizaciones participantes son microempresas (51.1\%) y pequeñas empresas (28.4\%); el $13.9 \%$ son empresas de tamaño medio y solamente el $6.6 \%$ son grandes empresas.

\section{Estudio de la eficacia de los planes de formación profesional en el ámbito laboral}

Como se ha indicado, uno de los objetivos principales de este estudio se centra en determinar el conocimiento que poseen las empresas del subsistema de FPE, del CNCP y de los CP como acreditación oficial dentro del ámbito laboral. Como muestra la figura 1 , la mayor parte de las empresas $(66.5 \%)$ no tienen conocimiento de la existencia de este subsistema, por lo que no saben cuál es su función y cómo pueden beneficiarse de las medidas que a partir del mismo se están aplicando. En la cuestión referida al CNCP, también la mayoría de los y las participantes (65.6\%) han informado no saber que es el Catálogo ni su utilidad. Finalmente, en lo que respecta a los CP, de nuevo la mayoría (59.8\%) contesta negativamente a la cuestión.

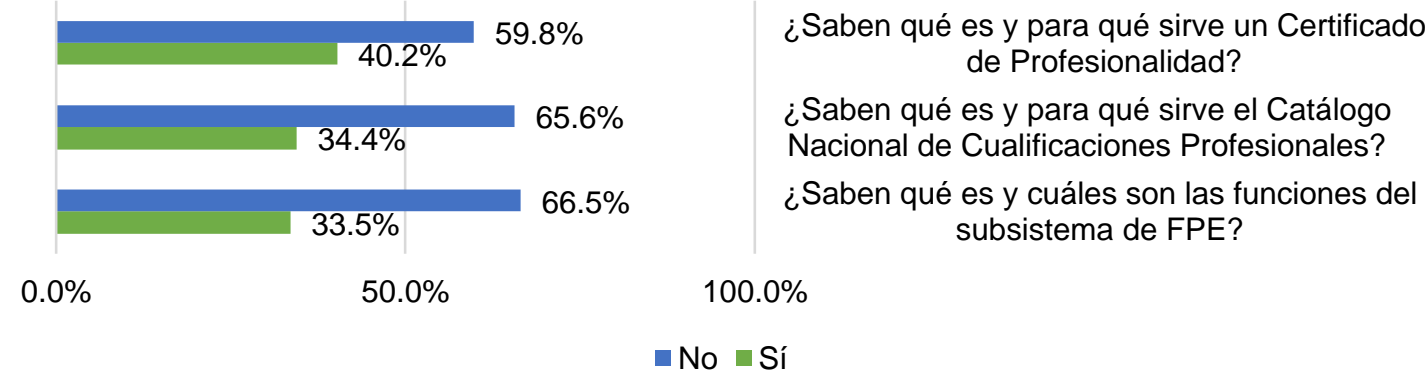

Figura 1. Conocimiento del subsistema de FPE, del CNCP y de los CP

Estos resultados permiten afirmar que, en general, hay un importante desconocimiento en cuanto al subsistema de FPE por parte de las empresas. No se sabe en qué consiste la FPE, qué es y para qué sirve un $\mathrm{CP}$, que existe un proceso de reconocimiento de las competencias profesionales o qué son las competencias clave.

Por otra parte, a aquellas empresas que han indicado que sí saben lo qué es y para qué sirve un $\mathrm{CP}$, se le dirigió a la cuestión referida a si tienen o han tenido contratado a algún trabajador/a que tuviese un CP, es 
decir, que realizase formación para su obtención o superase un proceso de reconocimiento de la experiencia laboral y aprendizajes no formales. En esta cuestión, también la mayoría $(84.21 \%)$ de las empresas han respondido negativamente.

Adicionalmente, se les ha preguntado si tenían o habían tenido realizando el módulo de prácticas profesionales no laborales a algún alumno/a que cursase un CP relacionado con la actividad de su empresa. Ante esta cuestión, la mayoría de las empresas que han indicado que saben qué es y para qué sirve un $\mathrm{CP}$, reconoce haber tenido a algún alumno/a realizando el módulo de prácticas (ver figura 2).

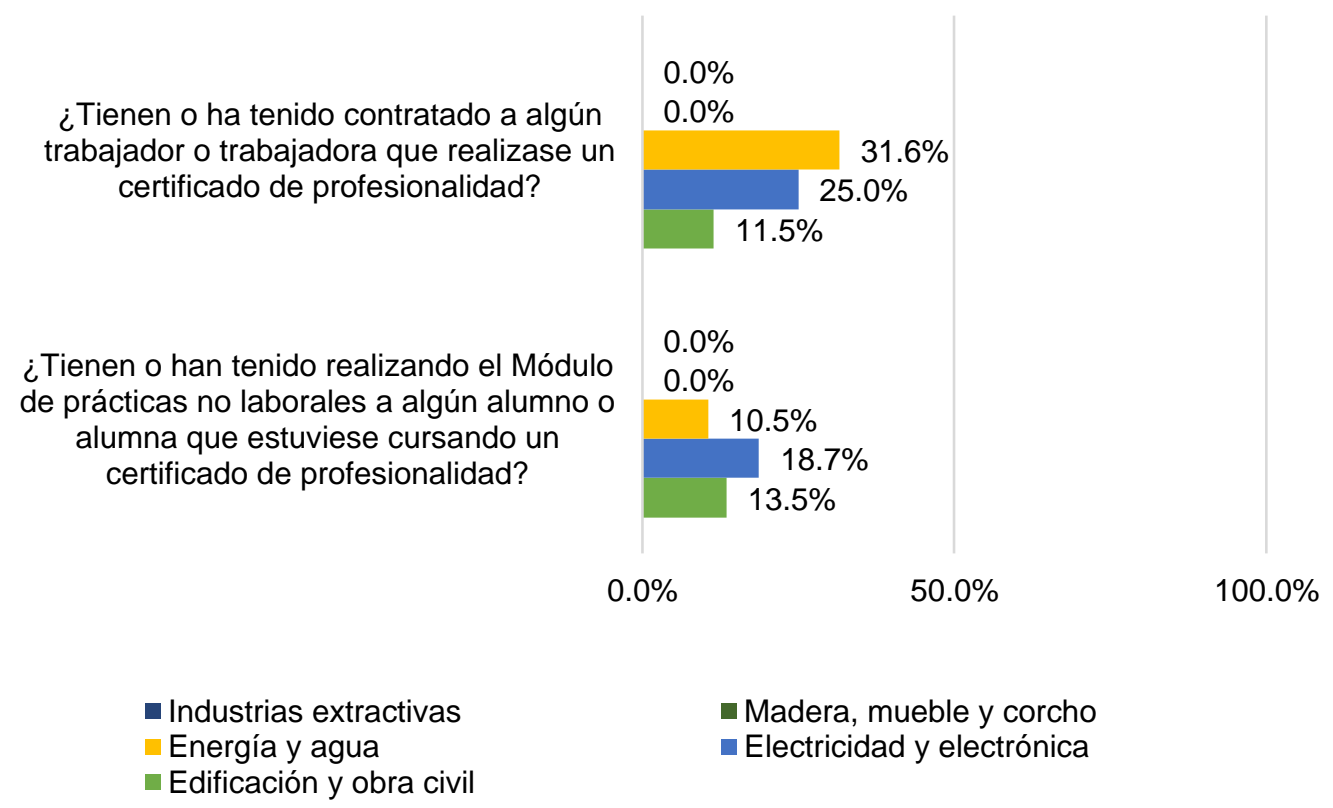

Figura 2. Contratación o acogida en prácticas de alumnado que realiza un CP

Así pues, los resultados nos permiten deducir que la mayoría de las empresas que conocen el subsistema de FPE, y qué son y para qué sirven los $\mathrm{CP}$, tienen este conocimiento debido a que han colaborado con centros y entidades de FPE en la acogida de alumnado durante el módulo de prácticas no laborales.

Por otro lado, a aquellas empresas que indicaron que conocían el subsistema, también se les ha preguntado si consideraban suficiente la oferta de FPE relacionada con las ocupaciones vinculadas a la actividad profesional de su empresa. Ante esta cuestión, el $81.98 \%$ ha señalado que la formación qué se oferta sí es suficiente para cubrir sus demandas actuales (ver figura 3), lo que informa que aquellos que conocen y han estado en contacto con el subsistema de FPE, están satisfechos con su organización y gestión.

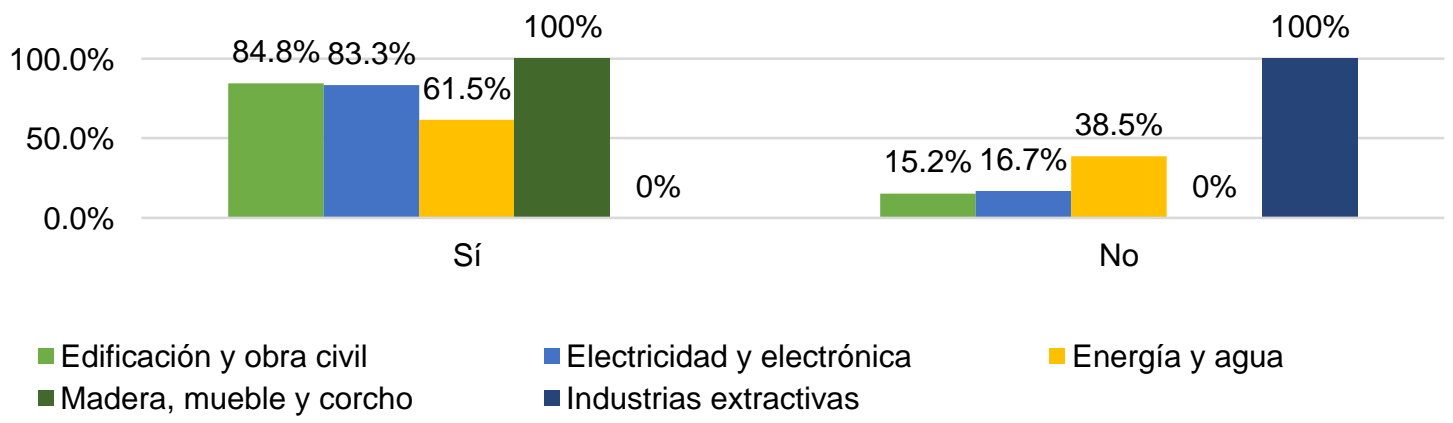

Figura 3. Considera suficiente la oferta de FPE relacionada con las ocupaciones vinculadas a la actividad profesional de su empresa 
En cuanto a la dimensión referida a las competencias transversales que demandan para sus trabajadores y trabajadoras, son muy valoradas por las empresas las siguientes: capacidad de trabajo en equipo, uso efectivo del tiempo, capacidad de resolución de problemas, compromiso, preocupación por la calidad y mejora, capacidad para la rápida adquisición de nuevos conocimientos, y capacidad de organización y planificación (ver tabla 4). No obstante, cabe señalar que la mayor parte de estas competencias son en general muy apreciadas por los participantes (entre 4.51 y 4.80 ). Las menos valoradas, sin dejar de considerarse igualmente necesarias, son el espíritu emprendedor y la mediación de conflictos (ambas 4.51).

\section{Tabla 4}

Competencias transversales (estadísticos descriptivos)

\begin{tabular}{cccc}
\hline Código & $\mathbf{N}$ & Media & DT \\
\hline C1 & 331 & 4.80 &, 398 \\
C2 & 331 & 4.80 &, 398 \\
C3 & 331 & 4.76 &, 471 \\
C4 & 331 & 4.77 &, 462 \\
C5 & 331 & 4.77 &, 462 \\
C6 & 331 & 4.75 &, 476 \\
C7 & 331 & 4.76 &, 471 \\
C8 & 331 & 4.76 &, 471 \\
C9 & 331 & 4.76 &, 471 \\
C10 & 331 & 4.75 &, 476 \\
C11 & 331 & 4.78 &, 428 \\
C12 & 331 & 4.78 &, 428 \\
C13 & 331 & 4.77 &, 462 \\
C14 & 331 & 4.77 &, 462 \\
C15 & 331 & 4.78 &, 428 \\
C16 & 331 & 4.51 &, 398 \\
C17 & 331 & 4.51 &, 398 \\
\hline
\end{tabular}

En cuanto al análisis diferencial comparativo de las necesidades de formación en competencias transversales según la familia profesional a la que pertenecen las empresas, después de aplicar la prueba Kruskal-Wallis para muestras independientes, podemos concluir que no existen diferencias significativas para las competencias transversales analizadas, ni tampoco ejecutando el análisis en función de la distribución geográfica.

Sin embargo, sí observamos diferencias dependiendo del tamaño de la empresa en las competencias C16 (mediación de conflictos) y C17 (espíritu emprendedor), con una significatividad en ambos casos de p=,001. Ambas competencias son más valoradas para el adecuado desempeño profesional en las microempresas $(65.08 \%)$ y pequeñas empresas $(64.89 \%)$, porcentaje que se reduce al $56.52 \%$ en las de tamaño medio y al $18.18 \%$ en las de gran tamaño. De esta forma, cuanto más pequeña es la empresa, más se estiman las competencias personales, las cuales aportan un valor añadido a la organización.

Sobre la percepción de las empresas en relación con la evolución del mercado de trabajo en su sector, una importante mayoría refiere que las perspectivas de empleo son negativas. Así, un $56.6 \%$ considera que progresivamente se producirá una notable reducción del empleo, frente a un $7.9 \%$ que indica que la tendencia será de crecimiento. El $36.6 \%$ restante piensa que la situación actual se mantendrá.

Según la familia profesional, los porcentajes obtenidos son muy similares. La mayoría de las empresas de las distintas familias se muestran pesimistas ante el futuro panorama (ver figura 4). 
$100.0 \%$

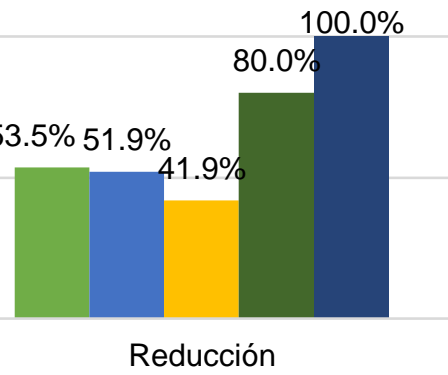

Edificación y obra civil

- Madera, mueble y corcho

- Electricidad y electrónica

- Energía y agua

- Industrias extractivas

Figura 4. Percepción sobre la evolución del mercado de trabajo (distribución por familias profesionales)

En lo que respecta a la percepción de los y las participantes sobre las nuevas demandas de formación generadas por los cambios en los contenidos o en las competencias de los puestos de trabajo de su sector, el $70.4 \%$ considera que es probable que surjan nuevas necesidades.

En función de la familia profesional los porcentajes también son similares (ver tabla 5). De este modo, las empresas de las distintas familias consideran que surgirán nuevas necesidades de formación generadas por los cambios en los contenidos o en las competencias de los puestos de trabajo.

\section{Tabla 5}

Percepción sobre nuevas necesidades de formación en función de la familia profesional

\begin{tabular}{|c|c|c|c|c|c|}
\hline & & \multicolumn{2}{|c|}{ Frecuencias } & \multicolumn{2}{|c|}{ Porcentajes } \\
\hline & & Sí & No & Sí & No \\
\hline \multirow{5}{*}{$\begin{array}{l}\text { Familia } \\
\text { profesional }\end{array}$} & Edificación y obra civil & 158 & 70 & 69.3 & 30.7 \\
\hline & Electricidad y electrónica & 33 & 19 & 63.5 & 36.5 \\
\hline & Energía y agua & 35 & 8 & 81.4 & 18.6 \\
\hline & Madera, mueble y corcho & 3 & 0 & 100 & 0 \\
\hline & Industrias extractivas & 4 & 1 & 80 & 20 \\
\hline \multirow{4}{*}{ Provincia } & A Coruña & 103 & 35 & 74.6 & 25.4 \\
\hline & Lugo & 34 & 24 & 58.6 & 41.4 \\
\hline & Ourense & 36 & 12 & 75 & 25 \\
\hline & Pontevedra & 60 & 27 & 69 & 31 \\
\hline \multirow{4}{*}{$\begin{array}{l}\text { Tamaño de } \\
\text { empresa }\end{array}$} & Microempresa & 100 & 69 & 59.2 & 40.8 \\
\hline & Pequeña empresa & 72 & 22 & 76.6 & 23.4 \\
\hline & Mediana empresa & 39 & 7 & 84.8 & 15.2 \\
\hline & Gran empresa & 22 & 0 & 100 & 0 \\
\hline
\end{tabular}

Acerca de la experiencia profesional previa, el $72.2 \%$ de las empresas manifestó considerarla imprescindible para el desempeño de las ocupaciones de su ámbito laboral. Por familias profesionales, provincias y tamaño de empresa, los resultados son muy similares, entendiendo la mayoría que se trata de un aspecto importante y necesario (ver tabla 6). 
Tabla 6

Percepción acerca de la importancia concedida a la experiencia profesional como factor clave

\begin{tabular}{|c|c|c|c|c|c|}
\hline & & \multicolumn{2}{|c|}{ Frecuencias } & \multicolumn{2}{|c|}{ Porcentajes } \\
\hline & & Sí & No & Sí & No \\
\hline \multirow{5}{*}{$\begin{array}{l}\text { Familia } \\
\text { profesional }\end{array}$} & Edificación y obra civil & 163 & 65 & 71.5 & 28.5 \\
\hline & Electricidad y electrónica & 33 & 19 & 63.5 & 36.5 \\
\hline & Energía y agua & 36 & 7 & 83.7 & 16.3 \\
\hline & Madera, mueble y corcho & 3 & 0 & 100 & 0 \\
\hline & Industrias extractivas & 4 & 1 & 80 & 20 \\
\hline \multirow{4}{*}{ Provincia } & A Coruña & 106 & 32 & 76.8 & 23,2 \\
\hline & Lugo & 36 & 22 & 62.1 & 37.9 \\
\hline & Ourense & 36 & 12 & 75 & 25 \\
\hline & Pontevedra & 61 & 26 & 70.1 & 29.9 \\
\hline \multirow{4}{*}{$\begin{array}{l}\text { Tamaño de } \\
\text { empresa }\end{array}$} & Microempresa & 103 & 66 & 60.9 & 39.1 \\
\hline & Pequeña empresa & 75 & 19 & 79.8 & 20.2 \\
\hline & Mediana empresa & 39 & 7 & 84.8 & 15.2 \\
\hline & Gran empresa & 22 & 0 & 100 & 0 \\
\hline
\end{tabular}

Como se ha puesto de manifiesto, existe un importante desconocimiento en cuanto al subsistema de FPE por parte de las empresas. Es más, los resultados informan que un importante porcentaje de estas tampoco conocen los CP como instrumento que acredita de forma oficial las cualificaciones profesionales del CNCP. Esta cuestión requiere de un profundo análisis -sobre todo considerando los recursos humanos y económicos que se destinan a este subsistema-, que a su vez ha de implicar una mayor promoción y reconocimiento de la FPE.

De igual forma, se antoja pertinente un estudio más exhaustivo sobre el desarrollo de las competencias transversales, que han de encontrarse incluidas de manera explícita en los diferentes $\mathrm{CP}$, sobre todo a la vista de su importancia para el mercado laboral. Una buena dirección la configurarían estudios sectoriales basados en el análisis de estas competencias pues, como se ha podido comprobar, el mundo de la empresa es plenamente consciente de que en los próximos años surgirán nuevas necesidades de formación generadas por los cambios en los contenidos o en las habilidades requeridas por los puestos de trabajo.

\section{Discusión}

Las acciones del subsistema de Formación Profesional para el Empleo tienen por objeto impulsar y extender entre las empresas y los trabajadores una formación que contribuya a la adquisición, mejora y actualización permanente de las competencias y cualificaciones profesionales, favoreciendo el aprendizaje a lo largo de la vida, y conjugando las necesidades de las personas, empresas, territorios y sectores productivos (Ley 56/2003, de 16 de diciembre, de Empleo, art. 26.2). No obstante, lo que se ha evidenciado es un profundo desconocimiento por parte de las organizaciones en cuanto a qué es, para qué sirve y cómo beneficiarse de todas las medidas que se aplican en el marco del subsistema de FPE, aun cuando este tiene entre sus objetivos dar respuesta a los requerimientos del mercado laboral. En este sentido, sería interesante comparar los resultados obtenidos con datos referidos a otras Comunidades Autónomas. Sin embargo, no es fácil encontrar evidencias de evaluación, desde el campo teórico e investigador, en torno a la formación profesional para el empleo (Marhuenda, 2012; Merino, 2012; OCDE., 2011; Ros, et al., 2017), y menos todavía de la Formación Ocupacional, que actualmente se corresponde, dentro de la FPE, con la modalidad de formación de oferta dirigida a personas trabajadoras desempleadas (De Miguel, San Fabian, Belver, \& Argüelles, 2011). Como también se ha podido comprobar en este trabajo, la actual sociedad del conocimiento demanda trabajadores con capacidad de reconversión y adaptación a los cambios, evitando quedarse al margen de una economía cada vez más global (Tito y Bill, 2016; Valero, 2012; Vázquez-Rodríguez, García-Álvarez, \& Santos Rego, 2021). Y es que, tal y como indica Vargas (2006), el sector productivo requiere de perfiles profesionales competitivos y multidisciplinares, donde las competencias de tipo transversal juegan un papel fundamental en la promoción de la empleabilidad. 
Son muchas las referencias que encontramos en la literatura al grupo de competencias que han sido objeto de análisis. Al respecto, Sutton (2002) afirma que estas competencias se constituyen como las más demandadas por las empresas de los diferentes sectores y áreas profesionales, señalando que son reconocidas por los/las empleadores/as como un factor clave para quienes aspiran a un puesto de trabajo. Sobre este particular, Cline (2005) identifica como más demandadas, las habilidades comunicativas, interpersonales y de aprendizaje, el deseo de promoción laboral dentro de la empresa, el trabajo en equipo y la resolución creativa de problemas. Puga y Martínez (2008) añaden a las anteriores las competencias vinculadas al liderazgo y la ética en el trabajo. Por su parte, Mitchel, Skinner, y White (2010) ponen el acento en la capacidad para gestionar el tiempo y de organización, la diversidad, el servicio al cliente y el pensamiento crítico. Robles (2012) incorpora la integridad, la cortesía, la responsabilidad, la actitud positiva y la flexibilidad. Y Hales (2012), asegura que el éxito en el trabajo del futuro se asienta sobre la base, no solo de destrezas técnico-profesionales, pues suponen en sí mismas un requisito, sino en competencias como el pensamiento crítico, resolución de problemas, habilidades comunicativas, o creatividad e innovación.

En esta línea, Queiruga (2018) y Rodríguez Sánchez (2002) subrayan la necesidad de un mayor ajuste entre formación de oferta y necesidades/demandas que suscita el mercado de trabajo. El modelo de empleo contemporáneo se caracteriza por una elevada movilidad laboral, donde las organizaciones buscan equipos de trabajo flexibles, altamente cualificados, con capacidad de adaptación a los cambios y habilidad para asumir reconversiones de forma continuada. Es por ello por lo que la formación de calidad no solo ha de considerar la adquisición de conocimientos, sino también el análisis de las necesidades emergentes en la búsqueda de una adecuada simbiosis entre el ámbito productivo y el educativo/formativo. Existe, por tanto, una valoración compartida que apuesta por la formación en competencias de carácter transversal, la cual identifica su desarrollo como factor clave del éxito en la promoción de la vida activa.

\section{Conclusiones}

Los prejuicios a los que se ha visto sometida la Formación Profesional están empezando a desaparecer. Hasta hace relativamente poco tiempo, se consideraba la única opción para aquellos alumnos y alumnas que no eran suficientemente "capaces" o "válidos", en cuanto a esfuerzo y responsabilidad, para acceder al sistema universitario. Sin embargo, actualmente, una buena parte de la sociedad ya es consciente de que la FP ofrece un itinerario formativo-profesional de calidad, y lo que es más importante, adaptado a las necesidades y demandas tanto del mercado laboral como de los trabajadores/as.

La alternativa de formación basada en el desarrollo de competencias transversales (saber, saber hacer y saber ser o estar) que ofrece la FP es verdaderamente interesante, ya que las empresas necesitan trabajadores/as capaces, no sólo de poner en práctica los conocimientos adquiridos, sino de adaptarse a los continuos cambios que nuestra sociedad experimenta.

En definitiva, constituye una imperiosa necesidad el proporcionar una mayor difusión a los recursos y oportunidades que brinda el subsistema de formación profesional para el empleo en lo referente a la diversidad de oferta formativa, medios, gratuidad, formación a demanda para las empresas, y acreditación de la cualificación de los trabajadores/as, entre otros. Empero, también habrá de considerar la oportunidad de introducir y orientar una dimensión competencial modulada por destrezas de tipo transversal en la planificación de los procesos de formación, contribuyendo al ajuste entre las esferas formativa y productiva.

\section{Referencias}

Acosta-Banda, A., Aguilar-Esteva, V., Patiño Ortiz, M., \& Patiño Ortiz, J. (2021). Contruction and Validity of an Instrument to Evaluate Renewable Energies and Energy Sustainability Perceptions for Social Consciousness. Sustainability, 13(4), 1-14. https://doi.org/10.3390/su13042333

Archer, W., \& Davison, J. (2008). Graduate Employability: What do employers think and want? The Council for Industry and Higher Education.

Bennett, R. (2002). Employers' Demands for Personal Transferable Skills in Graduates: a content analysis of 1000 job advertisements and an associated empirical study. Journal of Vocational Education and Training, 54(4), 457-476. https://doi.org/10.1080/13636820200200209

Blair, P. Q., \& Deming, D. J. (2020). Structural Increases in Demand for Skill after the Great Recession. AEA Papers and Proceedings 2020, 110, 362-365. https://doi.org/10.1257/pandp.20201064

Bridgstock, R. (2009). The graduate attributes we've overlooked: enhancing graduate employability through career management skills. Higher Education Research \& Development, 28(1), 31-44. https://doi.org/10.1080/07294360802444347 
Bunk, G. (1994). La transmisión de las competencias en la formación y perfeccionamiento profesionales de la RFA. Revista Europea de Formación Profesional, 1, 8-14. https://doi.org/10.30827/digibug.38089

Buendía, L., \& Berrocal, E. (2008). Evaluación de un programa de Formación Ocupacional para la inserción laboral. Revista de Investigación Educativa, 26(1), 157-190. https://doi.org/10.7203/relieve.12.1.4243

Buendía, L., Expósito, J., \& Sánchez, M. (2012). Investigación evaluativa de formación profesional para el empleo en el ámbito local. Revista de Investigación Educativa, 30(1), 161-179. https://doi.org/10.6018/rie.30.1.115971

Calvo, R., Payá, R., \& Sigalat, E. (2021). Cuando el aprendizaje basado en la práctica profesional no provoca uninterés laboral. Revista Fuentes, 23(1), 77-90. https://doi.org/10.12795/revistafuentes.2021.v23.i1.12024

Casal, J., Colomé, F., \& Comas, M. (2003). La interrelación de los tres subsistemas de formación profesional en España. Fundación Tripartita para la Formación en el Empleo. https://bit.ly/3ppDZu7

CEDEFOP. (2014). Terminology of European education and training policy. https://bit.ly/3cNayOZ

Cline, S. (2005). Soft skills make the difference in the workplace. Colorado Springs Business Journal. Recuperado de https://bit.ly/3EKAyGg

Chisvert, M. J., \& Marhuenda, F. (2012). Transiciones tempranas al mercado de trabajo. RASE. Revista de la Asociación de Sociología de la Educación, 5(2), 153-168. https://doi.org/10.7203/rase.11.2.12531

Construmatica. (2020). Metaportal de arquitectura, ingeniería y construcción. https://bit.ly/3cP0TIF

Cronbach, L. J. (1951). Coefficient alpha and the internal structure of tests. Psychometrika, 16, $297-334$. https://doi.org/10.1007/bf02310555

De Miguel, M., San Fabian, J. L., Belver, J. L., \& Argüelles, M. C. (2011). Evaluación de la satisfacción de los participantes en la formación profesional para el empleo. RELIEVE. Revista Electrónica de Investigación y Evaluación Educativa, 17(1), 1-30. https://doi.org/10.7203/relieve.17.1.4120

Deming, D. J. (2017). The Growing Importance of Social Skills in the Labor Market. The Quartely Journal of Economics, 132(4), 1593-1640. https://doi.org/10.1093/qie/qjx022

Fernández-García, A., García Llamas, J. L., \& García Pérez, M. (2019). La formación profesional básica, una alternativa para atender las necesidades educativas de los jóvenes en riesgo social. Revista de Humanidades, 36, $211-232$. https://doi.org/10.5944/rdh.36.2019.22564

Hales, S. (2012). Skills for tomorrow. American Management Association.

Instituto Galego de Estatística. (2020). Empresas con actividade en Galicia segundo actividade principal (grupos CNAE 2009) e condición xurídica. https://bit.ly/3eKT31I

Izquierdo, M., Puente, S., \& Font, P. (2013). Evolución del desajuste educativo entre la oferta y la demanda de trabajo en España. Banco de España. Boletín Económico, 43-50. https://bit.ly/3pjZ60V

Lorente, R. (2011). La reforma de la formación profesional en España a través del diseño por competencias: un análisis desde la perspectiva de los agentes sociales. Tesis doctoral: Universidad de Granada. https://bit.ly/2Zie7WC

Marhuenda, F. (2012). La Formación Profesional. Síntesis.

Martínez-Clares, P., González-Lorente, C., \& Rebollo-Quintela, N. (2019). Competencias para la empleabilidad: un modelo de ecuaciones estructurales en la Facultad de Educación. Revista de Investigación Educativa, 37(1), 57-73. https://doi.org/10.6018/rie.37.1.343891

Merino, R. (2012). La formación profesional en los itinerarios formativos y laborales de los jóvenes: las leyes proponen y los jóvenes disponen. Revista de la Asociación de Sociología de la Educación, 5(3), 503-512.

Ministerio de Educación y Formación Profesional. (2020). La Formación Profesional para el Empleo en el ambito laboral. https://bit.ly/3jSh6P6

Ministerio de Educación y Formación Profesional. (2021). Marco Español de Cualificaciones. https://bit.ly/3qm7aQ6

Mitchel, G., Skinner, L. B., \& White, B. J. (2010). Essential Soft Skills for Success in the Twenty-First Century Workforce as Perceived by Business Educators. Delta Pi Epsilon Journal, 52(1), 43-53.

Observatorio de la Fundación Laboral de la Construcción. (2020). Barómetro. https://bit.ly/2MOyyHt

OCDE. (2011). Preparándose para trabajar. OECD Publishing. https://doi.org/10.1787/9789264118478-es

Olaz, A. (2011). Una aproximación conceptual a la cualificación profesional desde una perspectiva competencial. Papers: Revista de Sociología, 96(2), 589-616. http://dx.doi.org/10.5565/rev/papers/v96n2.154

Osuna, J. L., Bueno, C., Murciano, J., \& Porrini, L. (2010). Los niveles de evaluación y seguimiento de la formación: calidad vs. Política. Revista Fuentes, 10, 101-122. http://dx.doi.org/10.12795/revistafuentes

Pérez Esparrells, C., \& Rahona, M. (2009). La formación profesional en España y el mercado de trabajo: Desafíos y oportunidades. https://bit.ly/2qw3S1Y

Pineda, P. (Coord.) (2002). Pedagogía Laboral. Editorial Ariel.

Pineda-López, R. C., \& Moreno-Bastida, G. R. (2019). Instrumentos para la Determinación de los Factores de la Inserción Laboral en Estudiantes Universitarios. Revista Científica Hallazgos21, 4(2), 173-189. https://bit.ly/3pUmB1I

Puga, J., \& Martinez, L. (2008). Competencias Directivas en Escenarios Globales. Estudios Gerenciales, 24(109), 87103.

PwC España. (2014). Trabajar en 2033. https://pwc.to/2yD4JTr

Queiruga, O. (2018). O subsistema de Formación Profesional para o Emprego: os Certificados de Profesionalidade e a súa vinculación coa mellora da empregabilidade en Galicia. Tesis doctoral: Universidade de Santiago de Compostela. https://bit.ly/3cQOsMr 
Rial, A. (2002). El presente en la formación para el "trabajo": las rápidas mutaciones en las exigencias de cualificación. Educar, 0, 177-181. https://bit.ly/2OJ6eHG

Robles, M. (2012). Executive Perceptions of the Top 10 Soft Skills Needed in Today's Workplace. Business Communication Quarterly, 75(4), 453-465.

Rodríguez-Sánchez, M. (2002). Análisis del módulo de formación en centros de trabajo en la familia profesional administrativa en la Comunidad de Madrid. Universidad Complutense de Madrid.

Ros, A., Navas, A., \& Marhuenda, F. (2017). Teorías implícitas del profesorado de formación profesional para el empleo. Estudios Pedagógicos, 43(1), 269-288. https://doi.org/10.4067/s0718-07052017000100016

Servicio Público de Empleo Estatal. (2019a). Informe del Mercado de Trabajo Estatal. Datos 2018. https://bit.ly/2KWQ9cp

Servicio Público de Empleo Estatal. (2019b). Informe de Prospección y detección de necesidades formativas 2019. https://bit.ly/2VUWvxF

Sistema Nacional de Empleo. (2016). Plan anual de Evaluación de la calidad, impacto, eficacia y eficiencia del conjunto del subsistema de formación profesional para el empleo 2014. https://bit.ly/2m6CdQq

Soler, M. (2014). Una mirada sobre la educación. La formación profesional. Revista Padres y Maestros, 359, 41-44. https://doi.org/10.14422/pym.i359.y2014.008

Suleman, F. (2018). The employability skills of higher education graduates: insights into conceptual frameworks and methodological options. Higher Education, 76, 263-278. https://doi.org/10.1007/s10734-017-0207-0

Sutton, N. (2002). Why can't we all just get along? Computing Canada, 28(16), 1-20.

Tito, M. D., \& Bill, O. (2016). Desarrollo de soft skills una alternativa a la escasez de talento humano. INNOVA Research Journal, 1(12), 59-76. doi: https://doi.org/10.33890/innova.v1.12.2016.81

Valencia, M. (2005). El capital humano, otro activo de su empresa. Entramado, 1(2), 20-33. https://bit.ly/3pkbFt7

Valero, F. J. (2012). Estudio Demandas del Mercado Laboral a los Titulados Universitarios. Servicio de Publicaciones de la Universidad de Granada.

Vargas, F. (2006). De las virtudes laborales a las competencias clave: un nuevo concepto para antiguas demandas. Politécnica, 3, 13-26.

Vázquez-Rodríguez, A., García-Álvarez, J., \& Santos Rego, M. A. (2021). Movilidad internacional y empleabilidad: el impacto en el capital humano y social de la juventud. Educar, 57(1), 81-96. https://doi.org/10.5565/rev/educar. 1185

World Economic Forum. (2018). The Future of Jobs Report. 2018. Centre for the New Economy and Society. 